David C. Taylor-Robinson ${ }^{1}$, Karsten Thielen ${ }^{2}$, Tania Pressler ${ }^{3}$, Hanne V. Olesen ${ }^{4}$, Finn Diderichsen ${ }^{2}$, Peter J. Diggle ${ }^{5}$, Rosalind Smyth ${ }^{6}$ and Margaret Whitehead ${ }^{1}$

${ }^{1}$ Dept of Public Health and Policy, Whelan Building, University of Liverpool, Liverpool, UK. ${ }^{2}$ Dept of Social Medicine, University of Copenhagen, Copenhagen, Denmark. ${ }^{3}$ Cystic Fibrosis Center, Rigshospitalet, Copenhagen, Denmark. ${ }^{4}$ Paediatric Dept A, Cystic Fibrosis Center, Aarhus University Hospital, Aarhus, Denmark. ${ }^{5}$ Institute of Infection and Global Health, University of Liverpool, Liverpool, UK. ${ }^{6}$ UCL Institute of Child Health, London, UK.

Correspondence: David C. Taylor-Robinson, Dept of Public Health and Policy, Whelan Building, University of Liverpool, Liverpool, L69 3GB, UK. Email: dctr@liv.ac.uk

Received: April 042014 | Accepted after revision: July 062014 | First published online: Sept 032014

Support statement: This work was supported by a Medical Research Council Population Health Scientist Fellowship awarded to D.C. Taylor-Robinson (G0802448). The funders had no role in the study design, data collection and analysis, decision to publish, or preparation of the manuscript.

Conflict of interest: Disclosures can be found alongside the online version of this article at erj.ersjournals.com

\title{
References
}

Schechter MS, Shelton BJ, Margolis PA, et al. The association of socioeconomic status with outcomes in cystic fibrosis patients in the United States. Am J Respir Crit Care Med 2001; 163: 1331-1337.

2 O'Connor GT, Quinton HB, Kneeland T, et al. Median household income and mortality rate in cystic fibrosis. Pediatrics 2003; 111: e333-e339.

3 Taylor-Robinson D, Smyth RL, Diggle P, et al. The effect of social deprivation on clinical outcomes and the use of treatments in the UK cystic fibrosis population: a longitudinal study. Lancet Respir Med 2013; 1: 121-128.

4 Barr HL, Britton J, Smyth AR, et al. Association between socioeconomic status, sex and age at death from cystic fibrosis in England and Wales (1959 to 2008): cross sectional study. BMJ 2011; 343: d4662.

Taylor-Robinson D, Schechter MS. Health inequalities and cystic fibrosis. BMJ 2011; 343: d4818.

Taylor-Robinson D, Whitehead M, Diderichsen F, et al. Understanding the natural progression in \%FEV1 decline in patients with cystic fibrosis: a longitudinal study. Thorax 2012; 67: 860-866.

Miller MR, Hankinson J, Brusasco V, et al. Standardisation of spirometry. Eur Respir J 2005; 26: 319-338.

8 Wang X, Dockery DW, Wypij D, et al. Pulmonary function between 6 and 18 years of age. Pediatr Pulmonol 1993; 15: 75-88.

9 Hankinson JL, Odencrantz JR, Fedan KB. Spirometric reference values from a sample of the general U.S. population. Am J Respir Crit Care Med 1999; 159: 179-187.

10 Laird NM, Ware JH. Random-effects models for longitudinal data. Biometrics 1982; 38: 963-974.

11 Diderichsen F, Andersen I, Manuel C, et al. Health inequality - determinants and policies. Scand J Public Health 2012; 40: 12-105.

12 Taylor-Robinson DC, Smyth R, Diggle PJ, et al. A longitudinal study of the impact of social deprivation and disease severity on employment status in the UK cystic fibrosis population. PLoS One 2013; 8: e73322.

13 Schechter MS. Non-genetic influences on CF lung disease: the role of sociodemographic characteristics, environmental exposures and healthcare interventions. Pediatr Pulmonol Suppl 2004; 26: 82-85.

14 Greasemann H, Ratjen F. Early lung disease in cystic fibrosis. Lancet Respir Med 2013; 1: 148-157.

\section{The impact of novel tests for tuberculosis depends on the diagnostic cascade}

\section{To the Editor:}

At least 3 million people with active tuberculosis (TB) are missed by national systems every year. Reaching these individuals is a critical priority [1]. Novel molecular diagnostics, notably Xpert MTB/RIF (Cepheid Inc., Sunnyvale, CA, USA) [2, 3], are important tools in this effort. Over 6 million Xpert cartridges have been procured worldwide since late 2010 [4] but two recent randomised trials in southern Africa [5, 6] suggest that Xpert, despite high sensitivity, may not significantly reduce morbidity and mortality. It is therefore useful to demonstrate how TB diagnostics function not in isolation but rather as part of a "diagnostic cascade."

We therefore adapted a transmission model of diagnostic testing among adults with active TB in Southeast Asia [7]. This model categorises a high-burden population into subpopulations characterised by TB status, HIV status and access to TB care. Parameter values, available in the original publication, are consistent with 
other published models of TB $[8,9]$. For transparency, in this analysis, we did not consider multidrugresistant TB, public/private sector differences or urban/rural differences.

We modelled TB diagnosis as a series of care-seeking attempts occurring after a "pre-diagnostic" delay. Depending on the diagnostic test sensitivity, probability of empirical treatment and efficacy of therapy, each attempt ended either in recovery or return to the active infectious state. Our primary outcomes were 10year reductions in TB incidence and mortality if TB were diagnosed using Xpert (implemented immediately and fully throughout the population) versus sputum smear microscopy.

We fitted model parameters at the steady state to epidemiological data in India, including life expectancy (66 years [10]), adult HIV prevalence (0.3\% [11]), TB incidence (176 cases per 100000 per year [1]), mortality (14-32 deaths per 100000 per year [1]), proportion of incident cases that were previously treated (19\% [1]) and case detection proportion (59\% [1]). We assumed that all active TB starts as smear-negative and progresses over time, such that $25 \%$ of all prevalent TB is smear-positive [12].

We constructed six sequential scenarios, with each scenario incorporating one additional step in the diagnostic cascade. Each scenario was independently calibrated to the epidemiological characteristics above, after which a $2 \%$ annual decline in TB incidence was initiated, representing current trends. The scenarios were as follows. 1) Baseline: no pre-diagnostic period, population without access to care, Xpert machine failure, pre-treatment loss to follow-up or empiric treatment. 2) Pre-diagnostic period: addition of an initial 4.5-month pre-diagnostic delay [7] during which individuals are infectious but symptoms are insufficiently severe to prompt care-seeking. 3) Reduced access: further consideration that $15 \%$ of active TB patients may never access the system of "passive" TB diagnosis and treatment. 4) Mechanical difficulty: further consideration that $10 \%$ of Xpert machines might be nonfunctional due to mechanical failure, outstanding calibration or inconsistent electricity, thus requiring diagnosis by sputum smear. 5) Pre-treatment loss: further consideration that $15 \%$ of diagnosed patients are lost to follow-up before starting treatment [13]. 6) Empirical treatment: further consideration that a percentage of TB patients with negative smear or Xpert results start treatment without microbiological diagnosis (e.g. based on chest radiography or response to broad-spectrum antibiotics) but after a 1-month delay [5].

Without Xpert, TB incidence was projected to fall at 2\% annually, from 176 to 144 per 100000 per year over 10 years. In the baseline scenario, Xpert reduced TB incidence to 69.5 per 100000 per year (51\% reduction relative to diagnosis with smear) and mortality to 5 per 100000 per year (82\% reduction). Sequential incorporation of steps in the diagnostic cascade reduced the impact of Xpert (fig. 1), with projected 10-year reductions in incidence of $42 \%$ after including a 4.5 -month pre-diagnostic period, $33 \%$ after also accounting for people without access to care, $32 \%$ after incorporating mechanical difficulty and $27 \%$ after including pretreatment loss to follow-up. Corresponding reductions in mortality were $76 \%, 60 \%, 58 \%$ and $52 \%$.

Empirical diagnosis dramatically affected the projected impact of Xpert. Assuming $40 \%$ empirical treatment in the idealised baseline scenario blunted projected Xpert-associated reductions in incidence and mortality from $51 \%$ to $36 \%$ and $82 \%$ to $58 \%$, respectively. When added to the other elements of the diagnostic cascade, empirical treatment for $10 \%, 40 \%$ and $80 \%$ (as seen in the TB-NEAT trial [5]) of smear-negative TB cases attenuated the projected impact of Xpert from a $27 \%$ reduction in incidence to $23 \%, 12 \%$ and $3 \%$, respectively, with impact on mortality following a similar trajectory ( $7 \%$ reduction at $80 \%$ empiric treatment).

This model of TB diagnosis and transmission demonstrates that novel diagnostic tests including Xpert function as part of a diagnostic cascade. As such, the population-level impact of the same test, in the same population, can vary by over an order of magnitude. The impact of any new TB diagnostic test depends ultimately not on sensitivity, but on the effectiveness of a cascade containing the novel test compared to a cascade incorporating standard diagnostics and empiric therapy. Realistic diagnostic cascades include elements such as pre-diagnostic delay, reduced access, mechanical difficulties, pre-treatment loss to follow-up, and empirical treatment; each step sequentially reduces the impact of Xpert on TB morbidity and mortality.

These findings demonstrate that understanding the diagnostic cascade in any given locality is critical to identifying the appropriate role of novel diagnostics. Notably, this model represents a low-HIV-prevalence population, suggesting that empirical findings in HIV-endemic countries $[5,6]$ may not be restricted to areas with high HIV. In some settings (e.g. rural health outposts in low-income countries), empirical diagnosis is rare, such that novel diagnostics may have substantial impact; however, in these settings, earlier steps of the cascade (e.g. pre-diagnostic delays, mechanical difficulties and losses to follow-up) are more constraining. By contrast, other settings (e.g. urban middle-income countries) may have few losses in early steps of the cascade but have high empirical treatment levels (e.g. due to use of chest radiography and other supportive diagnostics), rendering novel diagnostic tests less effective. This analysis shows that high-quality data at all steps of the cascade, particularly empirical treatment practices, are necessary for accurate 


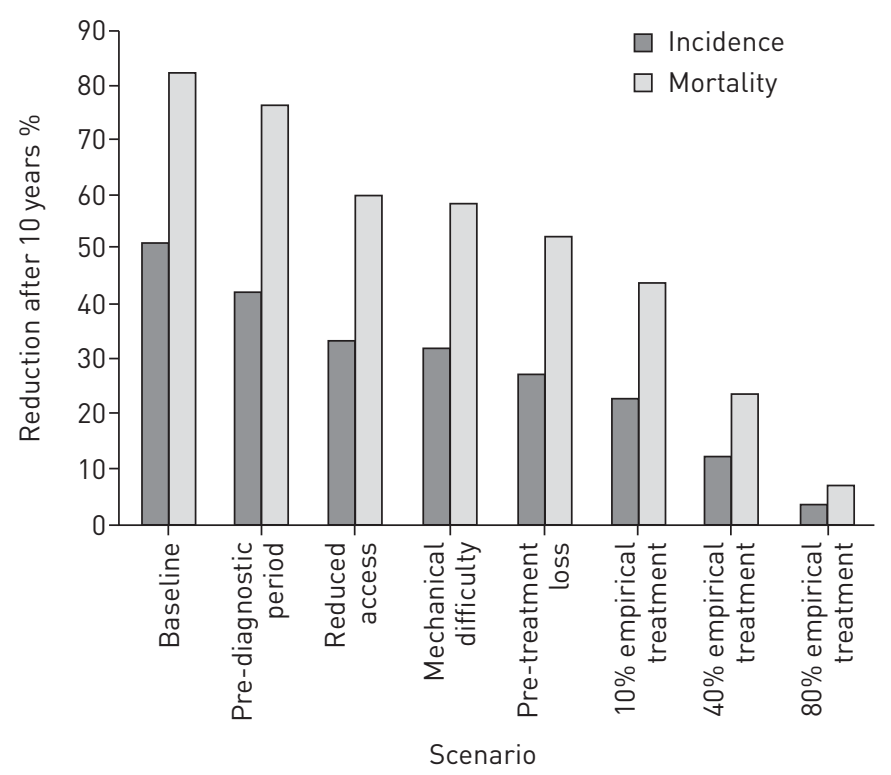

FIGURE 1 The diagnostic cascade in tuberculosis. Shown are the expected reductions in tuberculosis incidence and mortality 10 years after full replacement of sputum smear microscopy with Xpert MTB/RIF. Each sequential step to the right of the graph incorporates an additional element of tuberculosis diagnosis that may reduce the number of individuals benefiting from a more sensitive diagnostic test.

projections of local impact. Conversely, it also demonstrates the importance of quality assurance, linkage to care and provider confidence in a negative diagnostic test.

As with any transmission model, this model has limitations, including the assumption of homogeneous mixing. We did not account for public/private sector differences, HIV-induced immunosuppression or drug-resistant TB. Our depiction of the TB diagnostic cascade is incomplete; other steps (e.g. patient referral, communication of test results to providers/patients) may further limit diagnostic tests' impact. Ultimately, however, we aimed to demonstrate the importance of the diagnostic cascade, not to precisely project future trajectories in specific settings or any single step's role in that cascade.

In conclusion, $\mathrm{TB}$ diagnostic tests function as part of a larger system of diagnosis and treatment that we term the diagnostic cascade. Their impact in any given community therefore depends on the steps in that local cascade; the same test, deployed in different cascades, can spur a reduction in mortality ranging from nearly imperceptible to $>80 \%$. An understanding of these diagnostic cascades and the role of Xpert within them will be critical to the successful implementation of the World Health Organization's new TB Elimination framework $[14,15]$, now a priority in over 30 countries. In scaling up Xpert and other novel tests, greater attention must be paid to describing local TB diagnostic cascades and addressing factors that might limit their population-level impact.

0 @ERSpublications

The role of novel tests for TB in reducing morbidity and mortality depends on the system in which they are implemented http://ow.ly/Abrfo

Amanda Y. Sun ${ }^{1}$, Claudia M. Denkinger ${ }^{2,3}$ and David W. Dowdy ${ }^{4}$

${ }^{1}$ Johns Hopkins University School of Medicine, Baltimore, MD, USA. ${ }^{2}$ Foundation for Innovative New Diagnostics, Geneva, Switzerland. ${ }^{3}$ Beth Israel Deaconess Medical Center, Boston, MA, USA. ${ }^{4}$ Dept of Epidemiology, Johns Hopkins Bloomberg School of Public Health, Baltimore, MD, USA.

Correspondence: David W. Dowdy, Dept of Epidemiology, Johns Hopkins Bloomberg School of Public Health, 615 N. Wolfe St, E6531, Baltimore, MD, 21205, USA. E-mail: ddowdy@jhsph.edu

Received: June 182014 | Accepted after revision: July 282014 | First published online: Sept 032014

Support statement: This work was supported in part by the US National Institutes of Health (grants 1R21AI106031 and 5R21AI101152). C.M. Denkinger was supported by a fellowship from the Burroughs-Wellcome Fund from the American Society of Tropical Medicine and Hygiene. The funding sources had no role in study design, data collection and analysis, decision to publish, or preparation of the manuscript.

Conflict of interest: Disclosures can be found alongside the online version of this article at erj.ersjournals.com 


\section{References}

World Health Organization. Global tuberculosis report 2013. Geneva, WHO, 2013.

2 Pantoja A, Fitzpatrick C, Vassall A, et al. Xpert MTB/RIF for diagnosis of tuberculosis and drug-resistant tuberculosis: a cost and affordability analysis. Eur Respir J 2013; 42: 708-720.

3 Weyer K, Mirzayev F, Migliori GB, et al. Rapid molecular TB diagnosis: evidence, policy making and global implementation of Xpert MTB/RIF. Eur Respir J 2013; 42: 252-271.

4 World Health Organization. WHO monitoring of Xpert MTB/RIF roll-out. http://who.int/tb/laboratory/ mtbrifrollout/en/ Date last accessed: July 18, 2014.

5 Theron G, Zijenah L, Chanda D, et al. Feasibility, accuracy, and clinical effect of point-of-care Xpert MTB/RIF testing for tuberculosis in primary-care settings in Africa: a multicentre, randomised, controlled trial. Lancet 2014; 383: 424-435.

6 Churchyard G, McCarthy K, Fielding KL, et al. Effect of Xpert MTB/RIF on early mortality in adults with suspected TB: a pragmatic randomized trial. Top Antivir Med 2014; 22: Suppl. e-1, 48.

7 Sun AY, Pai M, Salje H, et al. Modeling the impact of alternative strategies for rapid molecular diagnosis of tuberculosis in Southeast Asia. Am J Epidemiol 2013; 178: 1740-1749.

8 Menzies NA, Cohen T, Lin HL, et al. Population health impact and cost-effectiveness of tuberculosis diagnosis with Xpert MTB/RIF: a dynamic simulation and economic evaluation. PLoS Med 2012; 9: e1001347.

9 Dye C, Garnett GP, Sleeman K, et al. Prospects for worldwide tuberculosis control under the WHO DOTS strategy. Lancet 1998; 352: 1886-1891.

10 World Bank. Life expectancy at birth, total (years). http://data.worldbank.org/indicator/SP.DYN.LE00.IN Date last accessed: April 9, 2014.

11 UNAIDS. UNAIDS report on the global AIDS epidemic 2012. Geneva, UNAIDS; 2012.

12 Gothi GD, Chakraborty AK, Nair SS, et al. Prevalence of tuberculosis in a south Indian district - twelve years after initial survey. Indian J Tuberc 1979; 26: 121-135.

13 Macpherson P, Houben RM, Glynn JR, et al. Pre-treatment loss to follow-up in tuberculosis patients in low- and lower-middle-income countries and high-burden countries: a systematic review and meta-analysis. Bull World Health Organ 2014; 92: 126-138.

14 Diel R, Loddenkemper R, Zellweger JP, et al. Old ideas to innovate tuberculosis control: preventive treatment to achieve elimination. Eur Respir J 2013; 42: 785-801.

15 D'Ambrosio L, Dara M, Tadolini M, et al. Tuberculosis elimination: theory and practice in Europe. Eur Respir J 2014; 43: 1410-1420.

\section{An evaluation of the use of a negative interferon- $\gamma$ release assay for tuberculosis screening before TNF antagonist therapy}

To the Editor:

Tumour necrosis factor (TNF)- $\alpha$ antagonists, such as infliximab, adalimumab and etanercept, are widely used to treat immune-mediated inflammatory diseases. These drugs increase the risk of latent infection with Mycobacterium tuberculosis (LTBI) reactivation [1, 2]. Tuberculosis (TB) preventative chemotherapy significantly reduces this risk (74\%); hence, in the UK, patients are screened for LTBI before starting TNF antagonist therapy [3]. The optimum screening strategy remains unclear. Strategies include combinations of clinical risk stratification data, T-cell interferon $-\gamma$ release assay (IGRA) tests and tuberculin skin tests (TSTs) $[2,4,5]$. These tests have limitations, and have discordant results when compared [6].

The 2005 British Thoracic Society (BTS) guidelines recommend the use of risk stratification tables based on population demographics. These balance the likelihood of LTBI with the risk of therapy-induced hepatotoxicity to guide preventative chemotherapy decisions [7].

Since 2005, IGRAs have become widely available and suggested as a TST alternative [8]. Guidelines have suggested that all IGRA-positive patients (unless secondary to previous, fully treated TB) be given preventative chemotherapy prior to commencing TNF antagonist therapy [9]. This generally consists of 6 months of isoniazid, or 3 months of rifampicin and isoniazid [10]. However, the management of IGRAnegative patients is more complex and this study focuses on this.

At our centre, patients with a negative IGRA (enzyme-linked immunospot assay) and normal chest radiography commence TNF antagonist therapy without referral for further LTBI risk assessment. This is 